\title{
CLINICAL EFFICACY OF SCIATIC NERVE BLOCK FOR BELOW-KNEE ORTHOPAEDIC SURGERIES
}

\author{
Swathi Hegde1, Manjunath H. G \\ 1 Postgraduate Student, Department of Anaesthesiology, Mysore Medical College and Research Institute, Mysore. \\ ${ }^{2}$ Associate Professor, Department of Anaesthesiology, Mysore Medical College and Research Institute, Mysore.
}

\section{ABSTRACT}

\section{BACKGROUND}

Sciatic nerve block is a useful technique for unilateral lower limb surgeries. Many studies with different approaches for sciatic nerve block have been conducted to demonstrate the efficacy of sciatic nerve block with different local anaesthetic drugs over the past decades. Ropivacaine, a long-acting amide local anaesthetic is found to be a safe and effective agent of choice for sciatic nerve block. Hence, this observational study was undertaken to evaluate the clinical efficacy of sciatic nerve block for intraoperative anaesthesia and post-operative analgesia in patients undergoing below-knee orthopaedic surgeries.

\section{MATERIALS AND METHODS}

After institutional ethical committee clearance and informed consent, 30 patients of either sex, between 18-60 years, ASA PS I and II, posted for elective lower limb surgeries were enrolled in the study. Using peripheral nerve stimulator, sciatic nerve block was given with $20 \mathrm{~mL}$ of $0.75 \%$ ropivacaine by classic approach of Labat. Onset of sensory and motor block, duration of analgesia, duration of motor block, quality of block, patient and surgeon satisfaction, adverse events and haemodynamic parameters were studied.

\section{RESULTS}

Onset of sensory and motor block were 21.74 minutes and 28.88 minutes respectively. Mean duration of analgesia at which rescue analgesic was given was 786.46 minutes. $96.66 \%$ surgeons rated the block as excellent and $93.33 \%$ patients had satisfactory block. There were no adverse events or haemodynamic instability.

\section{CONCLUSION}

We concluded that sciatic nerve block is an effective and safe method of anaesthesia for below-knee orthopaedic surgeries.

\section{KEYWORDS}

Ropivacaine, Sciatic Nerve Block.

HOW TO CITE THIS ARTICLE: Hegde S, Manjunath HG. Clinical efficacy of sciatic nerve block for below-knee orthopaedic surgeries. J. Evolution Med. Dent. Sci. 2016;5(87):6488-6491, DOI: 10.14260/Jemds/2016/1467

\begin{abstract}
BACKGROUND
Regional anaesthesia in the form of central neuraxial block has taken over as the principle technique for most of the lower limb surgeries However, over recent years, peripheral nerve blockade is increasingly becoming popular because of the additional advantages like.1,2,3, reduced incidence of adverse haemodynamic effects (hypotension, bradycardia), no postdural puncture headache, prolonged post-operative analgesia, less incidence of post-operative nausea and vomiting, reduced length of hospital stay. Sciatic nerve block is a useful technique for unilateral lower limb surgeries, particularly in patients considered unsuitable for central neuraxial block. ${ }^{4}$ Sciatic nerve block when combined with femoral nerve block provides effective anaesthesia of lower limb. It may also be used more readily in the presence of minor degree of coagulopathy or after head injury where central neuraxial
\end{abstract}

Financial or Other, Competing Interest: None.

Submission 12-10-2016, Peer Review 23-10-2016,

Acceptance 26-10-2016, Published 29-10-2016.

Corresponding Author:

Swathi Hegde,

D/o. Vishwanath Hegde,

"Susheela", Near C.T.O Complex,

Kagodu Thimmappa Nagar,

Sagar-577401,

Shimoga.

E-mail: swathihegde16@gmail.com

DOI: $10.14260 /$ jemds $/ 2016 / 1467$ block is relatively contraindicated. 4 Many studies with different approaches for sciatic nerve block have been conducted to demonstrate the efficacy of sciatic nerve block with different local anaesthetic drugs over the past decades. ${ }^{5}$ 11 Ropivacaine, a long-acting amide local anaesthetic is found to be safe and effective agent of choice for sciatic nerve block. 8,11

\section{Objectives}

To study the clinical efficacy of sciatic nerve block for intraoperative anaesthesia and post-operative analgesia in patients undergoing below-knee orthopaedic surgeries.

\section{MATERIALS AND METHODS}

After institutional ethical committee clearance and written informed consent, 30 patients aged 18 to 60 years of either sex, ASA Physical Status I and II, posted for elective below-knee orthopaedic surgeries were enrolled in the study. Patients with known hypersensitivity or contraindications to the study drugs, infection at the site of block, patients with severe renal, hepatic, respiratory or cardiac diseases, patients with severe coagulopathy, morbidly obese patients, pregnant patients, patients with neurological, psychiatric or neurovascular disorders, patient with alcohol/drug abuse and patients who refused the technique were excluded from the study. Parameters studied included onset of sensory blockade, onset of motor blockade, duration of analgesia, duration of motor 
blockade, quality of blockade, patient satisfaction, surgeon satisfaction, haemodynamic parameters and adverse effects.

A detailed pre-anaesthetic evaluation of each case was done previous day before surgery. A thorough systemic examination was carried out to detect the presence of any systemic disorder. Routine and special investigations were carried out accordingly. Local examination of block site was done to exclude any sign of sepsis, previous injury or previous deformity. Patients were kept nil orally for solids for 6 hours prior to surgery and Tab. Alprazolam $0.5 \mathrm{mg}$ and Tab. Ranitidine $150 \mathrm{mg}$ were given on the night prior to surgery. The patients were reassured, the procedure of block was explained and a written informed consent was obtained. On arrival of the patient in the operation theatre, Star plus of Larsen and Toubro multipara monitors were applied and base line respiratory rate, pulse rate, noninvasive blood pressure, SPO2 and ECG were recorded. Intravenous line was secured with 18G intravenous (IV) catheter and started on Ringer's lactate infusion. Premedication with Inj. Midazolam $1 \mathrm{mg}$ was given intravenously 5 minutes before the procedure.

All patients were positioned in Sims' position with the leg to be operated in nondependent position. Parts painted and draped. A line was drawn from the posterior superior iliac spine to the midpoint of the greater trochanter. A perpendicular line was drawn bisecting this line, which extended caudally. A second line was drawn from the greater trochanter to the sacral hiatus. The intersection of this line with the perpendicular line indicated the point of needle entry. After infiltrating the skin with $1 \%$ Lidocaine injection, the sciatic nerve was located using $10 \mathrm{~cm}$ insulated needle attached to peripheral nerve stimulator with the initial intensity of current set at $1.5 \mathrm{~mA}$. Needle placement was considered optimal when maximal plantar flexion of foot was obtained at a current of $<0.5 \mathrm{~mA}$, as plantar flexion of foot has got more success rate than dorsiflexion of foot. 12 After negative aspiration for blood, $20 \mathrm{~mL}$ of $0.75 \%$ ropivacaine was injected. Time of completion of injection was taken as time zero.

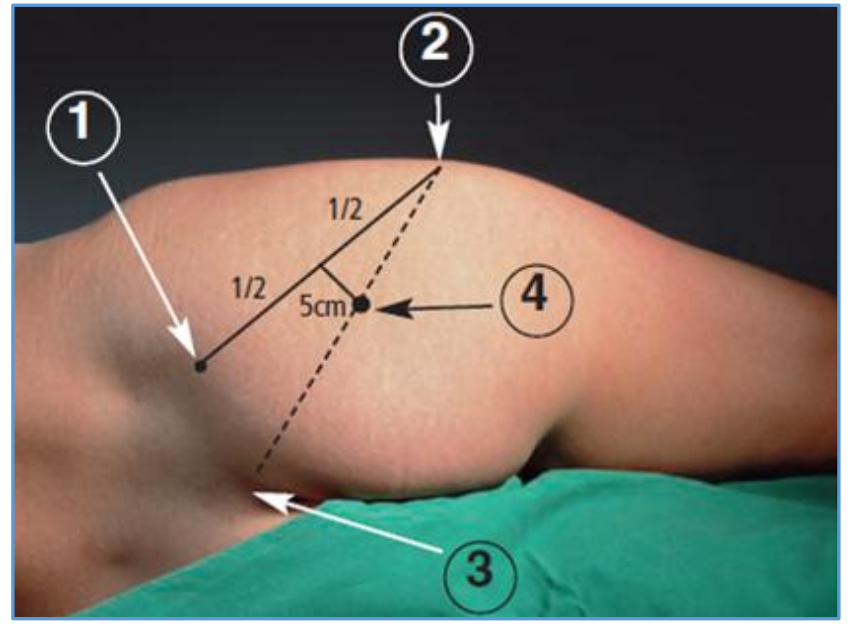

Image 1. Needle Entry Site for Sciatic Nerve Block

Immediately after the block placements, patients were assessed for sensory onset every minute by pinprick method on the dorsal and plantar aspects of the foot and sensation was categorised as ${ }^{4}$;

- $0=$ sharp (normal sensation as of contralateral limb).
- $1=$ dull (pinprick perceived as pressure).

- 2 =absent (complete loss of awareness of pinprick).

Motor block was assessed every minute for the onset by assessing plantar or dorsiflexion at the ankle and was graded as

- $0=$ normal power.

- $1=$ reduced power.

- $2=$ complete motor block.

At the end of 50 minutes, if patient did not develop sensory and motor anaesthesia, the block was considered inadequate ${ }^{13}$ and patients were given general anaesthesia. For statistical purposes, such patients were excluded.

As all the procedures were performed under standard thigh tourniquet, femoral nerve block was performed. After complete sensory and motor onset in the sciatic nerve distribution, patients were positioned supine. Under aseptic precautions, in supine position, femoral artery was palpated and the insulated needle was attached to peripheral nerve stimulator with the initial intensity of current set at $1 \mathrm{~mA}$. The needle was inserted $2 \mathrm{~cm}$ lateral to artery and $2 \mathrm{~cm}$ below the inguinal ligament. Needle placement was considered optimal when maximal quadriceps contraction or patellar snap was observed at a current of $<0.5 \mathrm{~mA}$. $15 \mathrm{~mL}$ of $2 \%$ lidocaine with 1:200,000 adrenaline was injected after negative aspiration for blood. After complete sensory onset, a standard thigh tourniquet was applied in all patients.

Throughout the surgery, haemodynamic parameters were recorded every 10 minutes. Patients were monitored for signs of local anaesthetic toxicity. Any hypersensitivity to drugs and other adverse reactions were noted.

In the post-operative period, resolution of motor blockade and duration of analgesia were recorded.

Patient satisfaction with the anaesthetic technique was recorded by asking the patient to assess the block as: very good, good, medium or poor. ${ }^{13}$ In the post-operative period, the pain was assessed by visual analogue score and at a score of 5, patients were given analgesic like Inj. Diclofenac $75 \mathrm{mg}$ and the study concluded at this point.

\section{Surgeon Satisfaction ${ }^{5}$ was assessed by Three-Point Score}

- $1=$ Completely satisfied. I want the same anaesthesia for future operations.

- $2=$ Partially satisfied. This anaesthesia may be adequate, but needs improvement.

- $\quad 3=$ Not satisfied. I want a different anaesthesia technique for future procedures.

- Quality of block was assessed using two point scale as follows ${ }^{13}$

- $\quad 0=$ Satisfactory (if block alone allowed surgery).

- $1=$ Unsatisfactory (need of analgesic supplementation or general anaesthesia to complete surgery).

- $\quad$ For statistical purpose, patients with unsatisfactory block were excluded from the study.

\section{RESULTS}

Mean age of the study population was 39.08 years. Mean weight was $58.9 \mathrm{~kg}$. Out of 30 patients, 17 patients were male and 13 patients were females. 26 patients belonged to ASA physical status (PS) I and rest of them belonged to ASA PS II. Mean duration of surgery was 43.8 minutes. 
The mean time for complete sensory onset was 21.74 minutes and the mean time for onset of complete motor block was 28.88 minutes. Mean duration of analgesia at which rescue analgesic was given was 786.46 minutes with a standard deviation of 259.65 minutes. Mean duration of motor block was 734.07 minutes. 28 patients out of 30 patients (93.33\%) had a satisfactory blockade. Two patients with unsatisfactory block were excluded from the study (6.66\%).

$17(60.71 \%)$ patients graded the block as very good, 5 (17.85\%) graded the experience as good, $6(21.4 \%)$ as medium and none of the patients graded as poor. $96.4 \%$ of surgeons rated the block as excellent.

All the patients remained haemodynamically stable throughout the procedure. There were no adverse events. Results were analysed using SPSS software.

\begin{tabular}{|c|c|c|}
\hline Mean Age & \multicolumn{2}{|c|}{39.08 Years } \\
\hline Mean weight & \multicolumn{2}{|c|}{58.9 KGS } \\
\hline \multirow{2}{*}{ Sex } & Male & Female \\
\cline { 2 - 3 } & 17 & 13 \\
\hline \multicolumn{2}{|c|}{ Table 1. Demographic Data } \\
\hline
\end{tabular}

\begin{tabular}{|c|c|}
\hline Parameters Observed & Mean Time in Minutes \\
\hline $\begin{array}{c}\text { Mean time for complete } \\
\text { sensory onset }\end{array}$ & 21.74 \\
\hline $\begin{array}{c}\text { Mean time for onset of } \\
\text { complete motor block }\end{array}$ & 28.88 \\
\hline $\begin{array}{c}\text { Mean duration of } \\
\text { analgesia }\end{array}$ & $\begin{array}{c}786.46 \text { (with a standard } \\
\text { deviation of 259.65 minutes) }\end{array}$ \\
\hline $\begin{array}{c}\text { Mean duration of motor } \\
\text { block }\end{array}$ & 734.07 \\
\hline \multicolumn{2}{|c|}{ Table 2. Block Parameters } \\
\hline \multicolumn{2}{|c|}{}
\end{tabular}

\begin{tabular}{|c|c|}
\hline Satisfactory Block & Unsatisfactory Block \\
\hline $28(93.33 \%)$ & $2(6.66 \%)$ \\
\hline \multicolumn{2}{|c|}{ Table 3. Quality of Block } \\
\hline
\end{tabular}

\begin{tabular}{|c|c|}
\hline Grading & Number of Patients \\
\hline Very good & $17(60.71 \%)$ \\
\hline Good & $5(17.86 \%)$ \\
\hline Medium & $6(21.4 \%)$ \\
\hline Poor & 0 \\
\hline \multicolumn{2}{|c|}{ Table 4. Patient Satisfaction } \\
\hline
\end{tabular}

\begin{tabular}{|c|c|}
\hline Grading & Number of Surgeons \\
\hline Completely satisfied & $27(96.4 \%)$ \\
\hline Partially satisfied & $1(3.6 \%)$ \\
\hline Not satisfied & 0 \\
\hline \multicolumn{2}{|c|}{ Table 5. Surgeon Satisfaction } \\
\hline
\end{tabular}

\section{DISCUSSION}

Lower limb nerve blocks are less popular than upper limb blocks due to wide spread acceptance, safety and reliability of central neuraxial block. However, interest in lower limb blocks has been increasing in view of prolonged pain relief and shorter hospital stay which are the two important goals of modern anaesthetic practice.
Other Advantages of Lower Limb Nerve Blocks Include:

1. Greater haemodynamic stability due to lack of sympathectomy, particularly suitable for low cardiac output states.

2. Unlike subarachnoid block, these techniques do not interfere with respiratory function, coughing or expectoration and allow dyspnoeic patient to sit upright in post-operative period with reduced risk of hypotension or syncope.

3. It can be applied in head injury and trauma patients, thus allowing monitoring of conscious level during surgery.

4. These blocks may be a safer alternative to central neuraxial block in the presence of minor degree of coagulopathy.

Sciatic nerve block is a useful technique of choice for below-knee orthopaedic surgeries and not many studies exist in evaluating its application for orthopaedic surgeries. Hence, we conducted an observational study to evaluate clinical efficacy of sciatic nerve block for intraoperative anaesthesia and post-operative analgesia in patients undergoing elective below-knee orthopaedic surgeries. Conolly ${ }^{4}$ et al concluded that $20 \mathrm{~mL}$ of either $0.5 \%$ bupivacaine or $0.75 \%$ ropivacaine produces effective anaesthesia and analgesia for foot surgeries and sciatic nerve block can be used as primary technique of choice in foot and ankle surgeries. Sinari $\mathrm{D}^{7}$ et al compared $0.5 \%$ bupivacaine and $0.75 \%$ ropivacaine for sciatic nerve block and reported less than 30 minutes for onset of surgical anaesthesia and duration of analgesia of 13 hours for bupivacaine and 16 hours for ropivacaine. Patient satisfaction was high among both the groups. Studies by Imbelloni et $\mathrm{al}^{9}$ concluded that combined femoro-sciatic block can be as effective as unilateral spinal and a safe alternative to spinal anaesthesia. Suzan Adali et al ${ }^{10}$ concluded that peripheral nerve block can be more advantageous in lower limb orthopaedic surgeries. Young $\mathrm{DS}^{14}$ et al conducted a retrospective cohort study on 78 consecutive patients who had a total ankle arthroplasty from October 2006 to June 2013. They concluded that infragluteal peripheral nerve block as a means of postoperative pain control in patients undergoing a total ankle replacement was associated with significantly decreased opioid use compared to patients receiving no peripheral nerve block. Monsó $\mathrm{A}^{15}$ et al reported no complications or sequelae after sciatic nerve blocks. They concluded that the technique was highly effective and comfortable for patients, as it required only one puncture and provided good postoperative analgesia with no major side effects.[15] Subhadra Arun Sinha ${ }^{16}$ et al concluded that single shot sciatic nerve block provided effective pain relief to majority of the patients up to 18 hours in below-knee orthopaedic surgery and also decreased analgesic drugs requirement postoperatively. SK Choudhary compared equal doses of clonidine and Dexmedetomidine with Levobupivacaine to study post-operative analgesia in femorosciatic nerve block in patients undergoing orthopaedic below-knee surgeries under subarachnoid block. They reported duration of analgesia up to 10 hours. ${ }^{17}$

The present study reported mean onset time for both sensory and motor block of less than 30 minutes and duration of analgesia up to 13 hours. We also observed a high satisfaction rate of above $90 \%$ among patients and surgeons. There were no adverse events or haemodynamic instability. 


\section{CONCLUSION}

With this study we conclude that the sciatic nerve block is an effective and safe method of anaesthesia for below-knee orthopaedic surgeries. It provides excellent intraoperative anaesthesia, haemodynamic stability and extended postoperative analgesia.

\section{REFERENCES}

1. Murray JM, Derbyshire S, Shields MO. Lower limb blocks. Anaesthesia 2010;65(1):57-66.

2. Jankowski CJ, Horlocker TT, Rock MJ, et al. Femoral 3-in-1 nerve block decreases recovery room time and charges and time to hospital discharge after outpatient knee arthroscopy. Reg Anesth Pain Med 1998;60:23S.

3. Kettner SC, Willschke H, Marhofer P. Does regional anaesthesia really improve outcome? British Journal of Anaesthesia 2011;107(S1):i90-5.

4. Connolly C, Coventry DM, Wildsmith JAW. Double blind comparison of ropivacaine $7.5 \mathrm{mg} \mathrm{ml}$ with bupivacaine 5 mg ml for sciatic nerve block. Br J Anaesth 2001;86(5):6747.

5. Casati A, Cappelleri G, Aldegheri G, et al. Total intravenous anesthesia, spinal anesthesia or combined sciatic-femoral nerve block for outpatient knee arthroscopy. Minerva Anestesiol 2004;70(6):493-502.

6. Beaulieu $P$, Babin $\mathrm{D}$, Hemmerling $T$. The pharmacodynamics of ropivacaine and bupivacaine in combined sciatic and femoral nerve blocks for total knee arthroplasty. Anesth Analg 2006;103(3):768-74.

7. Sinari D, Marino A, Chillemi S, et al. Sciatic nerve block with lateral popliteal approach for hallux valgus correction. Comparison between $0.5 \%$ bupivacaine and $0.75 \%$ ropivacaine. Minerva Anestesiologica 2004;70(9):625-9.

8. Fanelli G, Casati A, Beccaria $P$, et al. A double-blind comparison of ropivacaine, bupivacaine, and mepivacaine during sciatic and femoral nerve blockade. Anesth Analg 1998;87(3):597-600.

9. Imbelloni LE, Rezende GVP, Ganem EM, et al. Comparative study between combined sciatic femoral nerve block, via single injection, and spinal block anesthesia for unilateral surgery of lower limb. Rev Bras Anestesiol 2010; 60(6) :584-92, 324-8.
10. Adali S, Erkalp K, Erden V, et al. Spinal anaesthesia and combined sciatic nerve/lumbar plexus block techniques in lower extremity surgery. Acta Orthop Traumatol Turc 2011;45(4):225-32.

11. Casati A, Fanelli G, Borghi B, et al. Ropivacaine or mepivacaine $2 \%$ for lower limb peripheral nerve blocks. Study group on orthopedic anesthesia of the Italian society of anesthesia, analgesia, and intensive care. Anaesthesiology 1999;90(4):1047-52.

12. Taboada M, Atanassoff PG, Rodriguez J, et al. Plantar flexion seems more reliable than dorsiflexion with Labat's sciatic nerve block: a prospective randomized comparison. Anesth Analg 2005;100(1):250-4.

13. Cuvillon P, Nouvellon E, Ripart J, et al. A comparison of the pharmacodynamics and pharmacokinetics of bupivacaine, ropivacaine (with epinephrine) and their equal volume mixtures with lidocaine used for femoral and sciatic nerve blocks: a double-blind randomized study. Anesth Analg 2009;108(2):641-9.

14. Young DS, Cota A, Chaytor R. Continuous infragluteal sciatic nerve block for postoperative pain control after total ankle arthroplasty. Foot \& Ankle Specialist 2014;7(4):271-76.

15. Monso A, Santaliestra J, Barbal F, et al. Sciatic nerve block at the popliteal fossa for foot surgery. Revistaespanola De Anestesiologia Y Reanimacion 1996;43(1):27-9.

16. Sinha SA, Mutha SC, Phalgune DS. Efficacy of sciatic nerve block for pain management in below-knee orthopaedic surgery. Journal of Clinical and Diagnostic Research 2016;10(9):UC17-20.

17. Chaudhary SK, Verma RK, Rana S, et al. Ultrasound-guided femoro-sciatic nerve block for post-operative analgesia after below-knee orthopaedic surgeries under subarachnoid block: comparison between clonidine and dexmedetomidine as adjuvants to levobupivacaine. Indian J Anaesth 2016;60(7):484-90. 